\title{
Three-dimensional versus two-dimensional derived strain echocardiography for assessing right ventricular myocardial deformation in patients with chronic left ventricular heart failure: A proof-of- concept study
}

\section{Fei Gao}

Department of ultrasound, Second Affiliated Hospital of Harbin Medical University

Chong Liu

Department of Ultrasound, Second Affiliated Hospital of Harbin Medical University Qiang Guo

Department of Ultrasound, Jinshan Branch of Shanghai Sixth People's Hospital Affiliated of Shanghai Jiaotong University

\section{Shuang-quan Jiang}

Department of Ultrasound, Second Affiliated Hospital of harbin Medical University

\section{Zhen-zhen Wang}

Department of Ultrasound, Second Affiliated Hospital of Harbin Medical University

\section{Li Liu}

Department of Ultrasound, First People's Hospital of Heihe City

Hai-Ru Li

Department of Ultrasound, Second Affiliated Hospital of Harbin Medical University

\section{Guo-Qing Du}

Department of Ultrasound, Second Affiliated Hospital of Harbin Medical University Jia-wei Tian ( jwtian2004@163.com )

the Second Affiliated Hospital of Harbin Medical University https://orcid.org/0000-0002-5367-5402

Research article

Keywords: Echocardiography, right ventricular, three-dimensional, myocardial function, strain

Posted Date: September 3rd, 2020

DOI: https://doi.org/10.21203/rs.2.23108/v3 
License: (c) (i) This work is licensed under a Creative Commons Attribution 4.0 International License. Read Full License 


\section{Abstract}

Background: A novel three-dimensional echocardiography (3DE)-derived strain analysis software specialized for right ventricular (RV) monitoring is emerging that could definitely evaluate RV free wall and interventricular septum longitudinal strain. The aim of this study was to compare the diagnostic performance in evaluating RV function between 3DE and two-dimensional echocardiography (2DE)derived longitudinal strain.

Methods: Echocardiographic examinations were performed in 82 patients with RV dysfunction associated with chronic left-sided heart failure and 40 control subjects. RV dysfunction was defined as a $3 \mathrm{DE}$-derived RV ejection fraction (EF) $<45 \%$. Both $2 \mathrm{DE}$ and 3DE-derived strain analyses were performed in all the patients to measure the longitudinal strain of RV.

Results: 3DE-derived peak systolic longitudinal strain of RV free wall (RV-fwLS) was significantly lower in

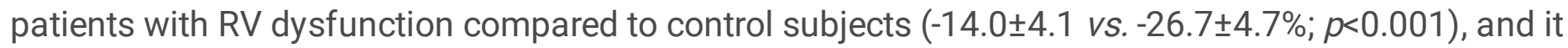
correlated well with cardiac magnetic resonance-derived RVEF $(r=0.74, p<0.001)$. On receiver operator characteristic analysis, a 3DE-derived RV-fwLS cutoff value of $>-21.1 \%$ was most useful in identifying patients at higher risk of RV dysfunction (sensitivity: $90 \%$ and specificity: $85 \%$ ), also higher than 2DEderived strain parameters. Additionally, RV dysfunctional patients with pulmonary hypertension $(\mathrm{PH})$ had significantly reduced 3DE-derived RV-fwLS value than the subgroup without $\mathrm{PH}(-13.1 \pm 3.8$ vs. $-15.0 \pm 4.2$; $p<0.05)$.

Conclusion区Assessment of impaired RV systolic function by 3DE-derived longitudinal strain is better than $2 D E$ in chronic left-sided heart failure patients with left ventricular $E F<45 \%$. 3DE-derived strain analysis specialized for RV should be considered as a complementary tool for assessing RV function.

\section{Background}

Assessment of right ventricular (RV) function has been validated as an important method for predicting clinical outcomes in patients with chronic left-sided heart failure, and even superior to left ventricular ejection fraction (LV-EF) [1, 2]. However, it is difficult to generate an accurate echocardiographic evaluation of RV myocardial function due to the complex anatomy and morphology involved [3]. Conventional echocardiographic parameters such as tricuspid annular plane systolic excursion (TAPSE) and RV fractional area change (FAC) are widely used in clinical practice. However, both of these methods have intrinsic shortcomings, which limit their specificity and reliability $[4,5]$.

Due to the longitudinal alignment of deep muscle fibers in RV, longitudinal shortening is a major contributor to RV systolic function. So longitudinal strain parameters can provide additional information about RV systolic function, enabling more accurate evaluation $[3,6,7]$. Many techniques have been introduced to quantitatively evaluate RV myocardial function by longitudinal strain. Two-dimensional (2D) speckle tracking echocardiography being used to evaluate LV systolic function, known for its ability to detect altered myocardial mechanics under different cardiovascular conditions, has already been 
applied to myocardial deformation assessments of RV [8-10]. However, RV deformation analyses based on two-dimensional echocardiography (2DE) are limited to be used in 2D planes in which volumetric assumption of the authentic morphology cannot be avoided.

Most recently, three-dimensional (3D) speckle tracking echocardiography originally developed for LV assessment was used to assess RV function with limitation in defining the location of the free wall-septal border $[9,12,13]$. The newly emerging three-dimensional echocardiography (3DE)-derived strain analysis particularly for RV have an evident advantage in its ability to recognize RV free wall and interventricular septum, is expected to provide more comprehensive assessment of RV systolic function [14].

In light of the potential technical advantages of this technology, we hypothesized that 3DE-derived longitudinal deformation parameters were more accurate than 2DE in assessing RV myocardial function. The aims of this study were to evaluate their diagnostic value in identifying patients with RV dysfunction as defined by the standard of 3DE-derived RVEF $<45 \%$ [4], and determined whether 3DE-derived longitudinal strain of RV correlates to cardiac magnetic resonance (CMR)-derived RVEF. Additionally we assessed whether there existed differences in the magnitude of 3DE-derived longitudinal deformation parameters of RV between RV dysfunctional patients with and without pulmonary hypertension $(\mathrm{PH})$.

\section{Methods}

The study complied with the Declaration of Helsinki and was approved by the Ethics Committee of Harbin Medical University. All patients signed a written informed consent form prior to participating in the study.

\section{Study Population}

From a large group of consecutive patients referred to our echocardiography center, 117 patients met with the diagnostic criteria of left-sided heart failure (LVEF $<45 \%)$ were recruited. Patients with atrial fibrillation, primary pulmonary hypertension, congenital heart disease or had undergone previous tricuspid valve surgery were excluded. Subjects with any obvious abnormalities of valvular structure or LV and/or RV geometry and function were also excluded. Sixteen (13.7\%) patients were excluded due to poor image quality (including poor acoustic windows, image artefacts, and enlarged RV being too large to fit entirely into the 3D full volume) or poor tracking.

An additional 40 healthy subjects with normal LV function, matched for gender and age, referred to our hospital for physical examination were prospectively enrolled as a control group. They volunteered to take routine examinations as needed, and were eligible as having no coronary artery disease documented by invasive coronary angiography or computed tomography angiography.

Finally, 82 patients with RV dysfunction (defined as 3DE-derived RVEF <45\%) and 40 healthy subjects were included. Among the patients with RV dysfunction, a cutoff value of $35 \mathrm{mmHg}$ [15] pulmonary pressure during systole was used to define $\mathrm{PH}$. Of the 82 patients, 40 (48.8\%) had PH and 42 (51.2\%) did not. 


\section{Traditional echocardiographic measurements}

A complete transthoracic echocardiogram was performed using a Vivid E9 scanner (GE Healthcare, Horten, Norway) in accordance with the guidelines for the examination of RV [4]. All echocardiographic measurements were processed according to the current guidelines [16]. The myocardial performance index (MPI) is a calculation based on tissue Doppler velocities from RV: (isovolumic relaxation time isovolumic contraction time) / RV ejection time. Conventional color Doppler imaging was used to grade the severity of tricuspid regurgitation semiquantitatively on a 4-point scale (normal, mild, moderate and severe) method by evaluating the ratio of tricuspid regurgitation jet area to right atrial area [17]. The systolic pulmonary pressure was assessed using tricuspid regurgitation peak velocity (performed by the modified Bernoulli equation) and right atrial pressure (estimated from the inferior vena cava diameter using a long-axis subxiphoid view and its response to inspiration).

\section{DE-derived strain analysis}

2DE-derived RV longitudinal strain analysis was offline assessed by the dedicated Echo-PAC version 201 software (GE Healthcare). Three consecutive heart cycles of modified apical 4-chamber images focus on RV were digitally saved for the subsequent analysis. Adjust the frequency, depth, and sector width to obtain the optimal frame rate of 60 to $90 \mathrm{frames} / \mathrm{s}$. All data was analyzed by an investigator who was blinded to the individuals' baseline clinical information. The investigator manually traced the endocardial contours and set the width to fit for the RV wall to obtain optimal tracking data. The region of interest consists of six segments (RV free wall and interventricular septum at three levels), which were automatically tracked frame by frame throughout the cardiac cycle. Peak systolic longitudinal strain of RV free wall (RV-fwLS) and peak systolic longitudinal strain of interventricular septum (sLS) were calculated by averaging peak systolic values in the three RV free wall segments or three interventricular septal segments, respectively.

\section{DE-derived strain analysis}

3D data were acquired with a fully sampled matrix-array V4 transducer. Consecutive 4-beat electrocardiographically triggered capture was acquired from a modified apical 4-chamber view focused on RV to enable generation of the full-volume data during breath hold. Special attention was paid to ascertain the pyramidal volume covered the entire RV myocardium with a scan angle as narrow as possible. Image optimization included adjustments of volume size, frame rate, gain, compression and depth. Mean volumetric frame rate was $21 \pm 6$ volumes/s in this study. The quality of the acquisitions was verified for each individual to ensure that optimal imaging had been done for the entire RV, without obvious stitching artifacts in the multislice display mode. 3D full-volume data were analyzed by a blinded investigator using the 4DAutoRVQ package (Echo-PAC version 201). Manual adjusted the sample lines to determine the axes of LV and RV chamber, the boundaries of aortic annulus and endocardium of RV in an end-diastolic frame of a quad view. Subsequently, three short-axis and one long-axis views of RV were generated to be used to manually delineate the detailed endocardium of RV in end-systole and enddiastole. The images that ultimately failed to track due to the suboptimal image quality were removed 
from the further analysis. The software then automatically traced the endocardial border and analyzed 3D tracking frame-by-frame, 3DE-derived volumetric parameters of RV, RVEF, RV-fwLS and RV-sLS were then generated. (Figure 1)

\section{CMR measurements}

CMR examinations were performed in 32 out of 82 patients with left-sided heart failure using a 1.5-T scanner (Siemens AG, Erlangen, Germany) with a phased-array cardiovascular coil. Eight to sixteen contiguous short-axis slices, paralleled to the plane of the atrioventricular valves, getting from the base to apex of the heart were obtained in each patient to generate the 2D steady-state free precession cine imaging. Slice thickness was dependent on patient size. RV volumetric analysis was then performed by two investigators blinded to the echocardiographic analysis. In each short-axis slice that encompassed the RV, the RV endocardial contour was manually traced at the end-diastolic frame and end-systolic frame. By the disc summation method, the RV end-diastolic volume (RVESV) and end-systolic volume (RVESV) were generated. RVEF was then calculated by the standard formula.

\section{Statistical analysis}

Statistical analyses were performed using Medcalc version 15.2. The normal distribution of each parameter was explored by the Kolmogorov-Smirnov test. Continuous data were expressed as the mean \pm standard deviation. Categorical data were expressed as numbers and percentages. The two groups were compared using independent student $t$-test, paired $t$-test, chi-square tests, Fisher's exact test or MannWhitney $U$ test depending on the type and distribution of the data. Correlations between two echocardiographic parameters were assessed with Spearman correlation coefficient. Receiver operating characteristic (ROC) curves and their area under the curve (AUC) were calculated to identify the diagnostic accuracy of 2DE and 3DE-derived RV deformation parameters for detection of patients with impaired RV systolic function. Then, we used the ROC curves to determine cutoff levels of corresponding sensitivity and specificity for the above-mentioned variables. AUC values were compared using the method proposed by DeLongs' [18]. Univariate and multivariate linear regression analyses were then performed to detect the independent correlates that would affect 3DE-derived RV-fwLS in the total study population. All parameters influencing the RV contractile function were selected for linear regression analysis; those parameters with $P$ values $<0.05$ in univariate analyses were entered into the multivariate model to complete the stepwise forward multiple regression analysis. Bland-Altman analysis was used to estimate mean intra-/interobserver differences from the corresponding repeated measurements, and reproducibility was assessed using intraclass correlation coefficients (ICCs) with $95 \%$ confidence intervals. $P<0.05$ was considered significant.

\section{Results}

\section{Baseline clinical characteristics}


As shown in table 1, both groups had similar age ranges (58.7 \pm 14.2 vs. $55.2 \pm 7.0, p=0.067)$ and sex distributions (male: $76.8 \%$ vs. $60.0 \%, p=0.054)$. In our study cohort, 46 (56.1\%) RV dysfunctional patients were concomitant with diabetes mellitus and/or hypertension. There were also no differences in clinical characteristics of all RV dysfunctional patients with and without performing CMR (table 2).

\section{Conventional echocardiographic parameters}

The table 3 listed all the values of conventional echocardiographic parameters. Compared to the controls, the patients with RV dysfunction had significantly lower TAPSE, RV-FAC and RV s' (all $p<0.001$ ). By contrast, the MPI were higher in RV dysfunction patients $(p<0.001)$. Among the patients with RV dysfunction, the differences of parameters, as the TAPSE and RV-FAC, were still significant between the subgroups with and without $\mathrm{PH}$ (all $p<0.05$ ). However, there was no statistical differences in the parameters of RV s' or MPI between these two subgroups (all $p>0.05$ ).

\section{Strain parameters}

Comparison of strain parameters between each two groups were summarized in Table 4. As seen in the table, the values of $3 D E$-derived RV-fwLS and SLS in the normal subjects were $-26.7 \pm 4.7 \%$ and $-15.5 \pm 4.3$ $\%$, respectively. The longitudinal strain of interventricular septum, using both 2DE and 3DE methods, were consistently lower than the corresponding values in free wall of RV for all the subjects (all $p<0.001$ ). As expected, the longitudinal strain values were severely reduced in patients with RV dysfunction than control group (all $p<0.001)$, take 3DE-derived RV-fwLS $(-14.0 \pm 4.1 v s$. $-26.7 \pm 4.7 \% ; p<0.001)$ for instance.

The RV dysfunction patients with PH had greater impairments of RV function assessed by 2DE and 3DEderived RV-fwLS than patients without $\mathrm{PH}$ (all $p<0.05$ ). Among the patients with RV dysfunction, we found that there existed no difference between subgroups with ischemia and nonischemic cardiomyopathy in the deformation parameters of 2DE- and 3DE-derived RV-fwLS $(-16.1 \pm 6.0 \mathrm{vs}$. $-15.2 \pm 6.9 \%,-14.4 \pm 4.3$ vs. $-13.6 \pm 3.7 \%$, all $p>0.05$ ). For the overall study population, we found that the values of 3DE-derived deformation parameters of RV showed relatively lower in magnitude than the corresponding 2DE-derived deformation parameters: RV-fwLS (-18.2 \pm 7.3 vs. $-19.4 \pm 7.9 \%, p=0.016), s L S$ $(-10.0 \pm 5.3$ vs. $-10.3 \pm 6.4 \%, p=0.377)$.

The figure 2 showed the ROC analyses of 2DE and 3DE- derived RV-fwLS to predict the patients with RV dysfunction. We detected that both 2DE and 3DE-derived RV-fwLS had good diagnostic accuracies for detection of patients with impaired RV dysfunction, while 3DE-derived RV-fwLS (AUC=0.975) showed higher predictive power than 2DE-derived RV-fwLS $(0.930)(p<0.05)$. With a cutoff value of $>-21.1 \%$ for 3DE-derived RV-fwLS presented optimal sensitivity of $90 \%$ and specificity of $85 \%$ to identify patients who are at a greater risk of RV dysfunction. On ROC analysis, we also discovered that 3DE-derived RV-fwLS had a good predictive ability to detect RV dysfunctional patients with $\mathrm{PH}(\mathrm{AUC}=0.638$ ), a cutoff value of $>-14.13 \%$ showed the best combination of sensitivity of $70 \%$ and specificity of $52 \%$. Moreover, $2 \mathrm{DE}-$ derived RV-fwLS (AUC=0.648) showed a relatively higher predictive performance than 3DE-derived RVfwLS, and the difference came to no significance $(p=0.882)$. 


\section{Correlation analysis}

The correlations of all the echocardiographic parameters to CMR-derived RVEF were presented in figure 3. We could know from the figure that all functional parameters were correlated significantly to CMR-derived RVEF $(p<0.001)$. Among the parameters of RV contraction, only 3DE-derived RV-fwLS showed a good correlation to CMR-derived RVEF $(r=-0.747, p<0.001)$. The correlation coefficient between 3DE-derived sLS and CMR-derived RVEF was moderate $(r=-0.458, p=0.008)$, weaker than 3DE-derived RV-fwLS. It was also found that 3DE-derived RV-fwLS presented higher correlations to CMR-derived RVEF than any of the following 2DE-derived deformation parameters: RV-fwLS ( $r=-527, p=0.002)$, sLS $(r=-0.366, p=0.039)$. Meanwhile, we discovered that the conventional echocardiographic parameters (as TAPSE, RV-FAC, RV s' or MPI) showed lower correlations to CMR-derived RVEF than either the 2DE or 3DE-derived deformation parameters of RV. As expected, 3DE-derived RVEF had strong correlation with CMR-derived RVEF $(r=0.855$, $p<0.001)$.

Significant univariate and multivariate correlates of 3DE-derived RV-fwLS in all the study subjects were shown in Table 5. The presence of diabetes, hypertension, hyperlipidemia, diminished LV end-diastolic volume (EDV), and lower TAPSE were all significant univariate associates of 3DE-derived RV-fwLS. Multivariate regression analysis using a stepwise forward algorithm demonstrated that combining with diabetes, hypertension, lower LVEDV and TAPSE were the independent determinants of 3DE-derived RVfwLS.

\section{Observer reproducibility}

Figure 4 exhibited the intraobserver and interobserver variability of measurements of 3DE-derived RV deformation parameters by Bland-Altman graphs. As presented in the figure, intra- and interobserver reproducibility was excellent for both 3DE-derived deformation parameters of RV (ICC >0.9). Intraobserver reproducibility for echocardiographic parameters of 3DE-derived RV-fwLS and SLS was 0.92 and 0.93 as assessed by ICC, and interobserver reproducibility for 3DE-derived RV-fWLS and sLS was 0.92 and 0.90, respectively. Intraobserver reproducibility for 2DE-derived RV-fwLS and SLS was 0.91 and 0.89 , and interobserver reproducibility for 2DE-derived RV-fwLS and SLS was 0.92 and 0.91 , respectively.

\section{Discussion}

The following conclusions were reached based on the study results evaluated above: 1) The 3DE-derived RV-fwLS was an appropriate parameter for predicting patients with impaired RV systolic function and more powerful than 2DE-derived RV-fwLS. 2) There were moderate to good correlations of 3D-derived RV longitudinal strain to CMR-derived RVEF, and the relation of 3DE-derived RV-fwLS to CMR-derived RVEF was greater than other measurements of RV systolic function. 3) RV dysfunctional patients with $\mathrm{PH}$ showed significant lower values in 3DE-derived RV-fwLS than patients without PH.

The RV performance is increasingly being recognized as a major predictor of long-term outcomes in various pathological conditions [19-21]. An analysis of RV performance based on geometric models in 
conventional 2DE method that could not assess the anterior and posterior walls or outflow tract of RV adequately, as these territories are poorly visualized in 2D planes. Given the irregular shape of RV, 3DEderived strain parameters could avoid this shortcoming to better reflect the true contractive condition of RV. To the best of our knowledge there is, as of yet, no published research assessing RV-fwLS in a 3DE environment for patients with RV dysfunction referred to chronic left-sided heart failure. We reported that 3DE-derived RV-fwLS has a better correlation to CMR-derived RVEF, and could detect more deformational features useful in identifying patients with RV dysfunction than 2DE-derived RV-fwLS. However, Smith et al. demonstrated 3DE-derived global longitudinal strain showed only moderate correlation to 3DE-derived RVEF, it might because of different system and vendor [13].

We observed that the correlation of 2DE or 3DE-derived RV longitudinal strain to CMR-derived RVEF was moderate to good. The RV myocardium mainly consists of myocardial fibers with the epicardial circumferential to oblique alignment and the subendocardial longitudinal alignment [3]. Longitudinal contraction contributes more to RV systolic function than transverse shortening, accounting for nearly $80 \%$ of overall RV function. Therefore, the dominant RV myocardial contraction is longitudinal direction $[6,7]$. Our study also indicated that the magnitude of the longitudinal strain in patients with RV dysfunction was significantly reduced relative to control subjects. In the present study, the variables of RV end-diastolic pressure and tricuspid regurgitation were not taken into analysis of the predictors of 3DEderived RV-fwLS. In fact, RV strain parameters are less load-dependent [4]. Meanwhile, the reference values of 3DE-derived longitudinal strain of RV should be established in subsequent studies.

Both ventricles share the oblique fibers in the interventricular septum, which may contribute to their independent contractions [22]. Owing to this important physiologic coaction between LV and RV, the contractile performance of RV is closely linked to the global performance of LV. In our study population, all RV dysfunctional patients were associated with chronic left heart failure. This might weaken the diagnostic accuracies of the corresponding deformation parameters in interventricular septum to discover patients with RV dysfunction. Furthermore, the LV becomes more spherical and interventricular septal myocardial fibers become less oblique because of chronic left heart failure, reducing their mechanical advantage to reflect the longitudinal function. In contrast, the RV-fwLS could analyze the RV mechanism free of influence from LV, are expected to provide more information for the actually contractive condition of RV. In our opinion, it was not appropriate to include the interventricular septum for calculating the global longitudinal strain of RV, as its inclusion would inevitably taint the data for RV analysis.

Along with the findings by Giusca et al. [23] and Wald et al. [24], we also found that conventional RV measurements (such as TAPSE or FAC) had relatively lower correlations to CMR-derived RVEF than RV deformation parameters. TAPSE is easily limited by regional abnormalities in the RV free wall tethering or tricuspid regurgitation, and RV-FAC has a major shortcoming of inter-measurement variability caused by its high dependence on the specific imaging plane. It is obvious that underestimations of TAPSE and RVFAC could result from its load dependency [4]. Overestimation of TAPSE may be caused by an increasing variability of the apical rotation [5] or the influence of medical therapy on RV regional motion to interfere 
its measurements. Nevertheless, RV free wall longitudinal strain is theoretically angle-independent and less susceptible to be influenced by cardiac translational motion, and as such may provide more accurate information concerning RV deformation pattern. Carluccio et al. also discovered that 2DE-derived RVfwLS could provide incremental prognostic information in heart failure patients with reduced LVEF and preserved TAPSE [25].

In our study cohort, patients with RV dysfunction were divided into two subgroups (those with and without $\mathrm{PH}$ ) according to the aforementioned cutoff value. RV dysfunctional patients with $\mathrm{PH}$ presented with significantly lower magnitudes of longitudinal strain in RV free wall. Increased LV filling pressures could lead to increased pulmonary artery pressure and further impair RV function by increasing the afterload. Our findings supported that 2DE or 3DE-derived RV-fwLS could identify a more advanced stage of cardiopulmonary involvement in patients associated with chronic left heart disease. Prior studies have showed that 2DE-derived RV longitudinal strain could help to predict morbidity and mortality in patients with $\mathrm{PH}[15,20]$. We hope to explore the potential ability of 3DE-derived RV-fwLS to predict prognosis among these patients in our future studies.

\section{Limitations}

Several limitations of the current study should be noted. Firstly, this was a single-territory study only with a small number of subjects, so further studies with a larger amount of subjects are needed to confirm our preliminary findings. Secondly, CMR has been considered as the gold standard for determining RV volumes and RVEF, this modality is limited by its high expense and low availability. However, CMR examinations were not available to all the subjects in this study, we also discovered that there was no difference in the clinical and conventional echocardiographic baseline data between subgroups with and without taking this examination. Thirdly, a portion of subjects in our study had been diagnosed with diabetes mellitus or hypertension which could potentially affect RV function. While excluding these subjects from the comparison did not alter the analysis of RV functional parameters between RV dysfunctional patients and control subjects. Fourthly, we did not assess 3D-derived RV myocardial deformation in radial and circumferential directions in this study. In fact, 3DE-derived radial and circumferential strains of RV were not analyzed owing to methodological limitations of this technology. Longitudinal shortening of the RV has already been shown to be a more important contributor to the RV systolic function compared to circumferential shortening [26]. Fifthly, a small portion of patients with preserved RV function and chronic left heart failure were excluded from the further research, so the results were non suitable to this subgroup, and our further study will expand the sample size to testify the feasibility of 3DE-derived strain analysis in this subgroup. Finally, our study subjects featured different types of etiologies, a larger study population with a pure etiology will be required to validate our findings.

\section{Conclusions}

We discovered that impaired RV systolic function assessed by 3DE-derived longitudinal strain is better than 2DE in chronic left-sided heart failure patients with LVEF $<45 \%$. 3DE-derived RV-fwLS outperformed 
most of currently recommended variables and other deformation parameters to have the highest correlation to CMR-derived RVEF, and it also could provide additional information for detecting RV dysfunctional patients with secondary PH. Therefore, our findings supported that the new advent 3DEderived strain analysis specialized for RV could be a reliable complementary tool for echocardiographic assessment of RV systolic function more accurately, which might be further used to identify subclinical $\mathrm{RV}$ dysfunction patients that need additional clinical management.

\section{Declarations}

\section{Ethics approval and consent to participate}

The study complied with the Declaration of Helsinki and was approved by the Ethics Committee of Harbin Medical University. All patients signed a written informed onsent form prior to participating in the study.

\section{Consent for publication}

Not applicable.

\section{Availability of data and materials $\square$}

All data generated or analysed during this study are included in this published article.

\section{Competing interests $\square$}

The authors declare that they have no competing interests.

\section{Funding:}

Not applicable.

\section{Authors' contributions:}

Concept/design: Tian JW, Du GQ, Gao F and Liu C; Data collection: Gao F, Liu C, Guo Q, Jiang SQ, Wang ZZ, Liu L, Li HR, Du GQ and Tian JW; Data analysis/interpretation: Gao F, Liu C, Guo Q, Jiang SQ, Wang ZZ, Liu L, Li HR; Drafting article: Gao F and Liu C; Critical revision of article: Gao F and Liu C; Statistics: Liu C; Approval of article: Tian JW and Du GQ.

\section{Acknowledgements:}

Not applicable.

\section{Abbreviations}

2D - Two-dimensional 
3D - Two-dimensional

AUC - Area under the curve

CMR - Cardiac magnetic resonance

EDV - End-diastolic volume

EF - Ejection fraction

ESV - End-systolic volume

FAC - Fractional area change

ICCs - Intraclass correlation coefficients

LV - Left ventricular

MPI - Myocardial performance index

$\mathrm{PH}$ - Pulmonary hypertension

ROC - Receiver operating characteristic

RV - Right ventricular

RV-fwLS - Peak systolic longitudinal strain of RV free wall

$s^{\prime}$ - Peak systolic velocity of tricuspid annular

sLS - Peak systolic longitudinal strain of interventricular septum

TAPSE - Tricuspid annular plane systolic excursion

\section{References}

1. Guendouz S, Rappeneau S, Nahum J, Dubois-Randé JL, Gueret P, Monin JL, et al. Prognostic significance and normal values of 2D strain to assess right ventricular systolic function in chronic heart failure. Circ J. 2012;76:127-36.

2. Motoki H, Borowski AG, Shrestha K, Hu B, Kusunose K, Troughton RW, et al. Right ventricular global longitudinal strain provides prognostic value incremental to left ventricular ejection fraction in patients with heart failure. J Am Soc Echocardiogr. 2014;27:726-32.

3. Ho SY, Nihoyannopoulos P. Anatomy, echocardiography, and normal right ventricular dimensions. Heart. 2006;92 Suppl 1:i2-13. 
4. Rudski LG, Lai WW, Afilalo J, Hua L, Handschumacher MD, Chandrasekaran K, et al. Guidelines for the echocardiographic assessment of the right heart in adults: A report from the American Society of Echocardiography endorsed by the European Association of Echocardiography, a registered branch of the European Society of Cardiology, and the Canadian Society of Echocardiography. J Am Soc Echocardiogr. 2010;23(7):685-71.

5. Motoji Y, Tanaka H, Fukuda Y, Sano H, Ryo K, Sawa T, et al. Association of apical longitudinal rotation with right ventricular performance in patients with pulmonary hypertension: insights into overestimation of tricuspid annular plane systolic excursion. Echocardiography. 2016;33(2):207-15.

6. Jamal F, Bergerot C, Argaud L, Loufouat J, Ovize M. Longitudinal strain quantitates regional right ventricular contractile function. Am J Physiol Heart Circ Physiol. 2003;285:H2842-7.

7. Carlsson $\mathrm{M}$, Ugander $\mathrm{M}$, Heiberg $\mathrm{E}$, Arheden $\mathrm{H}$. The quantitative relationship between longitudinal and radial function in left, right, and total heart pumping in humans. Am J Physiol Heart Circ Physiol. 2007;293:H636-H644.

8. Fukuda Y, Tanaka H, Sugiyama D, Ryo K, Onishi T, Fukuya H, et al. Utility of right ventricular free wall speckle tracking strain for evaluation of right ventricular performance in patients with pulmonary hypertension. J Am Soc Echocardiogr. 2011;24:1101-1108.

9. Vitarelli A, Mangieri E, Terzano C, Gaudio C, Salsano F, Rosato E, et al. Three-dimensional echocardiography and 2D-3D speckle-tracking imaging in chronic pulmonary hypertension: Diagnostic accuracy in detecting hemodynamic signs of right ventricular (RV) Failure. J Am Heart Assoc. 2015;4(3):e001584.

10. Roşca M, Călin A, Beladan CC, Enache R, Mateescu AD, Gurzun MM, et al. Rightventricular remodeling, its correlates, and its clinical impact in hypertrophic cardiomyopathy. J Am Soc Echocardiogr. 2015;28(11):1329-38.

11. Meris A,Faletra F, Conca C, Klersy C, Regoli F, Klimusina J, et al. Timing and magnitude of regional right ventricular function: A speckle tracking-derived strain study of normal subjects and patients with right ventricular dysfunction. J Am Soc Echocardiogr. 2010;23(8):823-31.

12. Ozawa K, Funabashi N, Takaoka H, Tanabe N, Yanagawa N, Tatsumi K, et al. Utility of threedimensional global longitudinal strain of the right ventricle using transthoracic echocardiography for right ventricular systolic function in pulmonary hypertension. Int J Cardiol. 2014;174:426-430.

13. Smith BC, Dobson G, Dawson D, Charalampopoulos A, Grapsa J, Nihoyannopoulos P. Threedimensional speckle tracking of the right ventricle: toward optimal quantification of right ventricular dysfunction in pulmonary hypertension. J Am Coll Cardiol. 2014;64:41-51.

14. Tamborini G, Cefalù C, Celeste F, Fusini L, Garlaschè A, Muratori M, et al. Multi-parametric "on board" evaluation of right ventricular function using three-dimensional echocardiography: feasibility and comparison to traditional two-and three dimensional echocardiographic measurements. Int $\mathrm{J}$ Cardiovasc Imaging.2019;35:275-284.

15. Mutlak D, Lessick J, Carasso S, Kapeliovich M, Dragu R, Hammerman $\mathrm{H}$, et al. Utility of pulmonary hypertension for the prediction of heart failure following acute myocardial infarction. Am J Cardiol. 
2012;109:1254-1259.

16. Lang RM, Badano LP, Mor-Avi V, Afilalo J, Armstrong A, Ernande L, et al. Recommendations for cardiac chamber quantification by echocardiography in adults: an update from the American Society of Echocardiography and the European Association of Cardiovascular Imaging. Eur Heart J Cardiovasc Imaging. 2015;16(3):233-70.

17. Suzuki Y, Kambara H, Kadota K, Tamaki S, Yamazato A, Nohara R, et al. Detection and evaluation of tricuspid regurgitation using a real-time, two-dimensional color-coded, Doppler flow imaging system: comparison with contrast two-dimensional echocardiography and right ventriculography. Am J Cardiol. 1986;57:811-5.

18. Bland JM, Altman DG. Statistical methods for assessing agreement between two methods of clinical measurement. Lancet. 1986;1:306-10.

19. Joyce E, Kamperidis V, Ninaber MK, Katsanos S, Debonnaire P, Schalij MJ, et al. Prevalence and correlates of early right ventricular dysfunction in sarcoidosis and its association with outcome. J Am Soc Echocardiogr. 2016;29(9):871-8.

20. Shahar K, Darawsha W, Yalonetsky S, Lessick J, Kapeliovich M, Dragu R, et al. Time dependence of the effect of right ventricular dysfunction on clinical outcomes after myocardial infarction: Role of pulmonary hypertension. J Am Heart Assoc. 2016;11:5(7).

21. Fine NM, Chen L, Bastiansen PM, Frantz RP, Pellikka PA, Oh JK, et al. Outcome prediction by quantitativeright ventricular function assessment in 575 subjects evaluated for pulmonary hypertension. Circ Cardiovasc Imaging. 2013;6(5):711-21.

22. Buckberg GD; RESTORE Group. The ventricular septum: the lion of right ventricular function, and its impact on right ventricular restoration. Eur J Cardiothorac Surg. 2006;29 Suppl 1:S272-8.

23. Giusca S, Dambrauskaite V, Scheurwegs C, D'hooge J, Claus P, Herbots L, et al. Deformation imaging describes right ventricular function better than longitudinal displacement of the tricuspid ring. 2010;96:281-288.

24. Wald RM, Haber I, Wald R, Valente AM, Powell AJ, Geva T. Effects of regional dysfunction and late gadolinium enhancement on global right ventricular function and exercise capacity in patients with repaired tetralogy of Fallot. Circulation. 2009;119:1370-1377.

25. Carluccio E, Biagioli P, Alunni G, Murrone A, Zuchi A, Coiro S, Riccini C, Anna Mengoni A, D'Antonio A, Ambrosio G. Prognostic Value of Right Ventricular Dysfunction in Heart Failure With Reduced Ejection Fraction: Superiority of Longitudinal Strain Over Tricuspid Annular Plane Systolic Excursion. Circ Cardiovasc Imaging. 2018;11:e006894.

26. Kukulski T, Hübbert L, Arnold M, Wranne B, Hatle L, Sutherland GR. Normal regional right ventricular function and its change with age: a Doppler myocardial imaging study. J Am Soc Echocardiogr. 2000;13:194-204.

\section{Tables}


Table 1 Clinical baseline characteristics

\begin{tabular}{|c|c|c|c|}
\hline Parameters & RV dysfunction ( $n=82)$ & Control group $(n=40)$ & $P$ value \\
\hline Age, years & $58.7 \pm 14.2$ & $55.2 \pm 7.0$ & 0.067 \\
\hline Male, $n(\%)$ & $63(76.8)$ & $24(60.0)$ & 0.054 \\
\hline Heart rate, beats/min & $89.5 \pm 17.8$ & $72.1 \pm 7.5$ & $<0.001$ \\
\hline $\mathrm{BMI}, \mathrm{kg} / \mathrm{m}^{2}$ & $40.5 \pm 6.2$ & $37.6 \pm 5.2$ & 0.013 \\
\hline \multicolumn{4}{|l|}{ Risk factors, n(\%) } \\
\hline Diabetes mellitus & $18(22.0)$ & $0(0)$ & 0.001 \\
\hline Hypertension & $39(47.6)$ & $1(2.5)$ & $<0.001$ \\
\hline Hyperlipidemia & $20(24.4)$ & $0(0)$ & $<0.001$ \\
\hline Smoker & $30(36.6)$ & $7(17.5)$ & 0.031 \\
\hline \multicolumn{4}{|c|}{ New York Heart Association Functional class, n (\%) } \\
\hline I & $0(0)$ & - & - \\
\hline II & $4(4.9)$ & - & - \\
\hline III & $26(31.7)$ & - & - \\
\hline IV & $52(63.4)$ & - & - \\
\hline \multicolumn{4}{|c|}{ Clinical underlying etiology diagnosis of heart failure, $n(\%)$} \\
\hline Ischemic heart disease & $47(57.3)$ & - & - \\
\hline Cardiomyopathy & $25(30.5)$ & - & - \\
\hline Valvular heart disease & $6(7.3)$ & - & - \\
\hline Hypertension & $4(4.9)$ & - & - \\
\hline
\end{tabular}

$\mathrm{RV}=$ right ventricular; $\mathrm{BMI}=$ body mass index .

Values are mean \pm SD or $\mathrm{n}(\%) . P<0.05$ was considered significant. Statistical significance are in boldface type.

Table 2 Clinical baseline and echocardiographic parameters between RV dysfunctional patients with and without performing CMR 


\begin{tabular}{|llll|}
\hline Parameters & CMR $+(\mathbf{n}=\mathbf{3 2})$ & CMR $-(\mathbf{n}=\mathbf{5 0})$ & Pvalue \\
\hline Age, years & $55.7 \pm 15.0$ & $60.6 \pm 13.4$ & 0.129 \\
\hline Male, $\mathbf{n}(\%)$ & $26(81.3)$ & $37(74.0)$ & 0.488 \\
\hline Heart rate, beats/min & $89.2 \pm 15.5$ & $89.7 \pm 19.2$ & 0.893 \\
\hline BMl, kg/m ${ }^{2}$ & $41.2 \pm 5.9$ & $40.0 \pm 6.4$ & 0.013 \\
\hline Risk factors, $\mathbf{n}(\%)$ & & & \\
\hline Diabetes mellitus & $8(25.0)$ & $10(20.0)$ & 0.594 \\
\hline Hypertension & $14(43.8)$ & $25(50.0)$ & 0.580 \\
\hline Hyperlipidemia & $7(21.9)$ & $13(26.0)$ & 0.671 \\
\hline Smoker & $8(25.0)$ & $22(44.0)$ & 0.081 \\
\hline BNP, pg/ml & $8463.9 \pm 9477.5$ & $10421.6 \pm 14257.1$ & 0.495 \\
\hline LV functional parameters & & $195.4 \pm 77.5$ & 0.347 \\
\hline LVEDV, ml & $211.8 \pm 76.0$ & $132.5 \pm 62.2$ & 0.341 \\
\hline LVESV, ml & $146.8 \pm 72.0$ & $33.6 \pm 10.1$ & 0.155 \\
\hline LVEF, \% & $30.4 \pm 9.8$ & & \\
\hline
\end{tabular}

$\mathrm{CMR}=$ cardiac magnetic resonance; $\mathrm{RV}=$ right ventricular; $\mathrm{BMI}=$ body mass index; $\mathrm{BNP}=$ brain natriuretic peptide.

Values are mean $\pm \mathrm{SD}$ or $\mathrm{n}(\%) . P<0.05$ was considered significant. Statistical significance are in boldface type.

Table 3 Conventional echocardiographic parameters from all subjects 


\begin{tabular}{|c|c|c|c|}
\hline Parameters & RV dysfunction ( $n=82$ ) & Control group $(n=40)$ & $P$ value \\
\hline \multicolumn{4}{|l|}{ LV functional parameters } \\
\hline LVEDV, ml & $201.8 \pm 76.9$ & $91.2 \pm 18.1$ & $<0.001$ \\
\hline LVESV, ml & $138.1 \pm 66.1$ & $29.3 \pm 7.6$ & $<0.001$ \\
\hline LVEF, \% & $32.4 \pm 10.0$ & $67.8 \pm 5.8$ & $<0.001$ \\
\hline \multicolumn{4}{|l|}{ Right heart functional parameters } \\
\hline RV Basal diameter, mm & $34.7 \pm 6.7$ & $29.3 \pm 5.1$ & $<0.001$ \\
\hline RV Longitudinal diameter, mm & $73.7 \pm 9.7$ & $65.2 \pm 8.9$ & $<0.001$ \\
\hline TAPSE, mm & $15.1 \pm 3.3$ & $21.9 \pm 2.9$ & $<0.001$ \\
\hline RVEDA, $\mathrm{cm}^{2}$ & $19.8 \pm 5.5$ & $15.8 \pm 3.4$ & $<0.001$ \\
\hline RVESA, $\mathrm{cm}^{2}$ & $14.2 \pm 5.3$ & $8.7 \pm 2.5$ & $<0.001$ \\
\hline RVFAC, \% & $29.6 \pm 12.5$ & $45.8 \pm 7.2$ & $<0.001$ \\
\hline $\mathrm{RV} \mathrm{S}^{\prime}, \mathrm{cm} / \mathrm{s}$ & $9.9 \pm 3.0$ & $12.3 \pm 2.0$ & $<0.001$ \\
\hline MPI, \% & $44.8 \pm 8.2$ & $38.9 \pm 8.0$ & $<0.001$ \\
\hline RA diameter, mm & $33.8 \pm 7.2$ & $30.9 \pm 4.7$ & 0.021 \\
\hline RVEDV, ml & $62.7 \pm 26.5$ & $39.6 \pm 11.1$ & $<0.001$ \\
\hline RVESV, ml & $43.5 \pm 19.7$ & $17.8 \pm 6.6$ & $<0.001$ \\
\hline RVEF, \% & $31.5 \pm 6.4$ & $53.4 \pm 4.9$ & $<0.001$ \\
\hline Tricuspid regurgitation & & & $<0.001$ \\
\hline No, n(\%) & $0(0)$ & $4(10.0)$ & \\
\hline Mild, $n(\%)$ & $28(34.1)$ & $36(90.0)$ & \\
\hline Moderate, $\mathrm{n}(\%)$ & $10(12.2)$ & $0(0)$ & \\
\hline Severe, $n(\%)$ & $44(53.7)$ & $0(0)$ & \\
\hline
\end{tabular}

$\mathrm{RV}=$ right ventricular; $\mathrm{LV}=$ left ventricular; $\mathrm{EDV}$ = end-systolic volume; $\mathrm{ESV}=$ end-diastolic volume; $\mathrm{EF}$ = ejection fraction; TAPSE = tricuspid annular plane systolic excursion; EDA = end-systolic area; ESA = enddiastolic area; $F A C=$ fractional area change; $s^{\prime}=$ peak systolic velocity of tricuspid annular; $\mathrm{MPI}=$ myocardial performance index; $\mathrm{RA}=$ right atrium.

Values are mean $\pm \mathrm{SD}$ or $\mathrm{n}(\%) . P<0.05$ was considered significant. Statistical significance are in boldface type. 
Table 4 Strain parameters

\begin{tabular}{|c|c|c|c|c|c|c|}
\hline \multirow[t]{3}{*}{ Parameters } & \multicolumn{3}{|l|}{ All subjects } & \multicolumn{3}{|c|}{ RV dysfunction } \\
\hline & $\begin{array}{l}\text { RV dysfunction ( } n= \\
\text { 82) }\end{array}$ & $\begin{array}{l}\text { Control } \\
\text { group }\end{array}$ & $\begin{array}{l}P \\
\text { value }\end{array}$ & $\begin{array}{l}\text { Without } \\
\text { PH }\end{array}$ & $\mathrm{PH}$ & $\begin{array}{l}P \\
\text { value }\end{array}$ \\
\hline & & $(n=40)$ & & $(n=42)$ & 40) & \\
\hline \multicolumn{7}{|c|}{ 2DE-derived strain parameters, $\%$} \\
\hline $\begin{array}{l}\text { 2DE-derived RV- } \\
\text { fwLS }\end{array}$ & $-15.7 \pm 6.3$ & $-27.1 \pm 4.8$ & $<0.001$ & $\begin{array}{l}-17.3 \pm \\
7.1\end{array}$ & $\begin{array}{l}-14.0 \pm \\
5.1\end{array}$ & 0.020 \\
\hline 2DE-derived SLS & $-6.8 \pm 4.1$ & $-17.6 \pm 3.4$ & $<0.001$ & $\begin{array}{l}-7.5 \pm \\
4.8\end{array}$ & $\begin{array}{l}-6.0 \pm \\
3.0\end{array}$ & 0.097 \\
\hline \multicolumn{7}{|c|}{ 3DE-derived strain parameters, \% } \\
\hline $\begin{array}{l}\text { 3DE-derived RV- } \\
\text { fwLS }\end{array}$ & $-14.0 \pm 4.1$ & $-26.7 \pm 4.7$ & $<0.001$ & $\begin{array}{l}-15.0 \pm \\
4.2\end{array}$ & $\begin{array}{l}-13.1 \pm \\
3.8\end{array}$ & 0.036 \\
\hline 3DE-derived SLS & $-7.3 \pm 3.3$ & $-15.5 \pm 4.3$ & $<0.001$ & $\begin{array}{l}-7.6 \pm \\
3.6\end{array}$ & $\begin{array}{l}-6.9 \pm \\
2.9\end{array}$ & 0.300 \\
\hline
\end{tabular}

$\mathrm{RV}=$ right ventricular; $\mathrm{PH}=$ pulmonary hypertension; $2 \mathrm{DE}$ = two-dimensional echocardiography; $3 \mathrm{DE}=$ three-dimensional echocardiography; RV-fwLS = peak systolic longitudinal strain in free wall of right ventricule; $S L S=$ peak systolic longitudinal strain in ventricular septum.

Values are mean \pm SD. $P<0.05$ was considered significant. Statistical significance are in boldface type.

Table 5 Univariate and multivariate linear analysis for 3DE-derived RV-fwLS

in all the study subjects 


\begin{tabular}{|llllll|}
\hline Variable & \multicolumn{3}{l}{ Univariate } & \multicolumn{3}{l|}{ Multivariate } \\
\hline & $\boldsymbol{R}$ & $\mathbf{9 5 \%} \mathbf{C l}$ & $\boldsymbol{P}$ & $\mathbf{9 5 \%} \mathbf{C l}$ & $\boldsymbol{P}$ \\
\hline Age & 0.11 & $-0.067-0.284$ & 0.219 & - & - \\
\hline BMI & 0.18 & $-0.001-0.344$ & 0.051 & - & - \\
\hline Diabetes & 0.33 & $0.159-0.477$ & $<0.001$ & $0.100-0.439$ & $\mathbf{0 . 0 0 3}$ \\
\hline Hypertension & 0.38 & $0.218-0.523$ & $<0.001$ & $0.043-0.391$ & $\mathbf{0 . 0 1 6}$ \\
\hline Hyperlipidemia & 0.23 & $0.059-0.395$ & $\mathbf{0 . 0 0 9}$ & $-0.099-0.265$ & 0.363 \\
\hline Smoking & 0.16 & $-0.021-0.326$ & 0.083 & - & - \\
\hline LVEDV & 0.58 & $0.445-0.685$ & $<0.001$ & $0.299-0.591$ & $<0.001$ \\
\hline TAPSE & -0.71 & -0.786 to -0.605 & $<0.001$ & -0.693 to -0.449 & $<0.001$ \\
\hline
\end{tabular}

All abbreviations are as shown in Table 1 or Table 2.

$P<0.05$ was considered significant. Statistical significance are in boldface type.

\section{Figures}




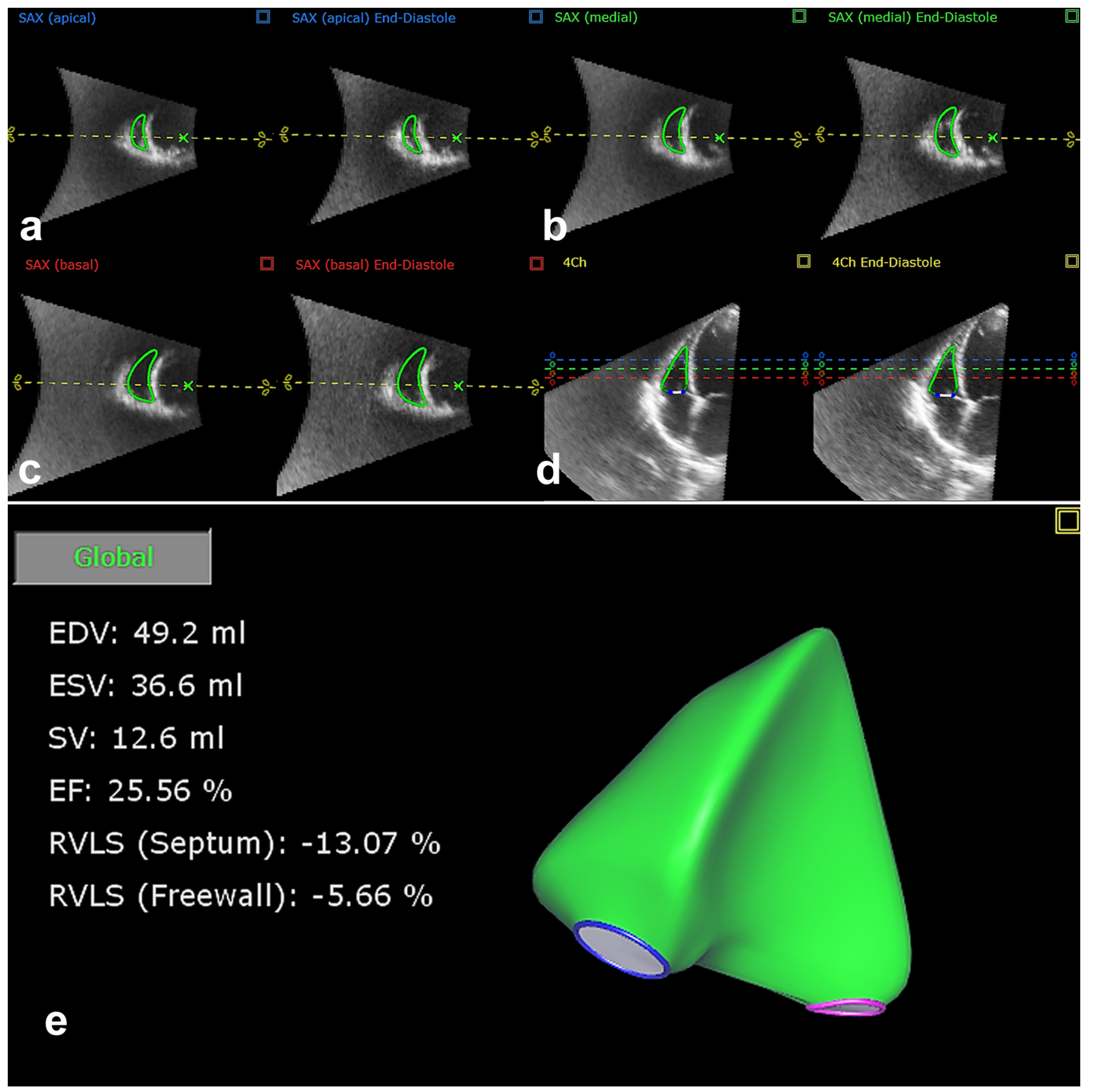

Figure 1

Example of 3DE-derived strain analysis for RV in a patient with RV dysfunction (a-d) manual tracing the true RV endocardial border in three short and one longitudinal views during end-diastole; (e) 3DE-derived $\mathrm{RV}$ reconstruction image seen in front view with listed corresponding contractive function parameter values. $3 \mathrm{DE}=$ three-dimensional echocardiography; $\mathrm{RV}=$ right ventricular; $E D V=$ end-diastolic volume; $\mathrm{ESV}$ = end-systolic volume; $\mathrm{SV}$ = stroke volume; $\mathrm{EF}$ = ejection fraction; RVLS, peak systolic longitudinal strain of right ventricle. 


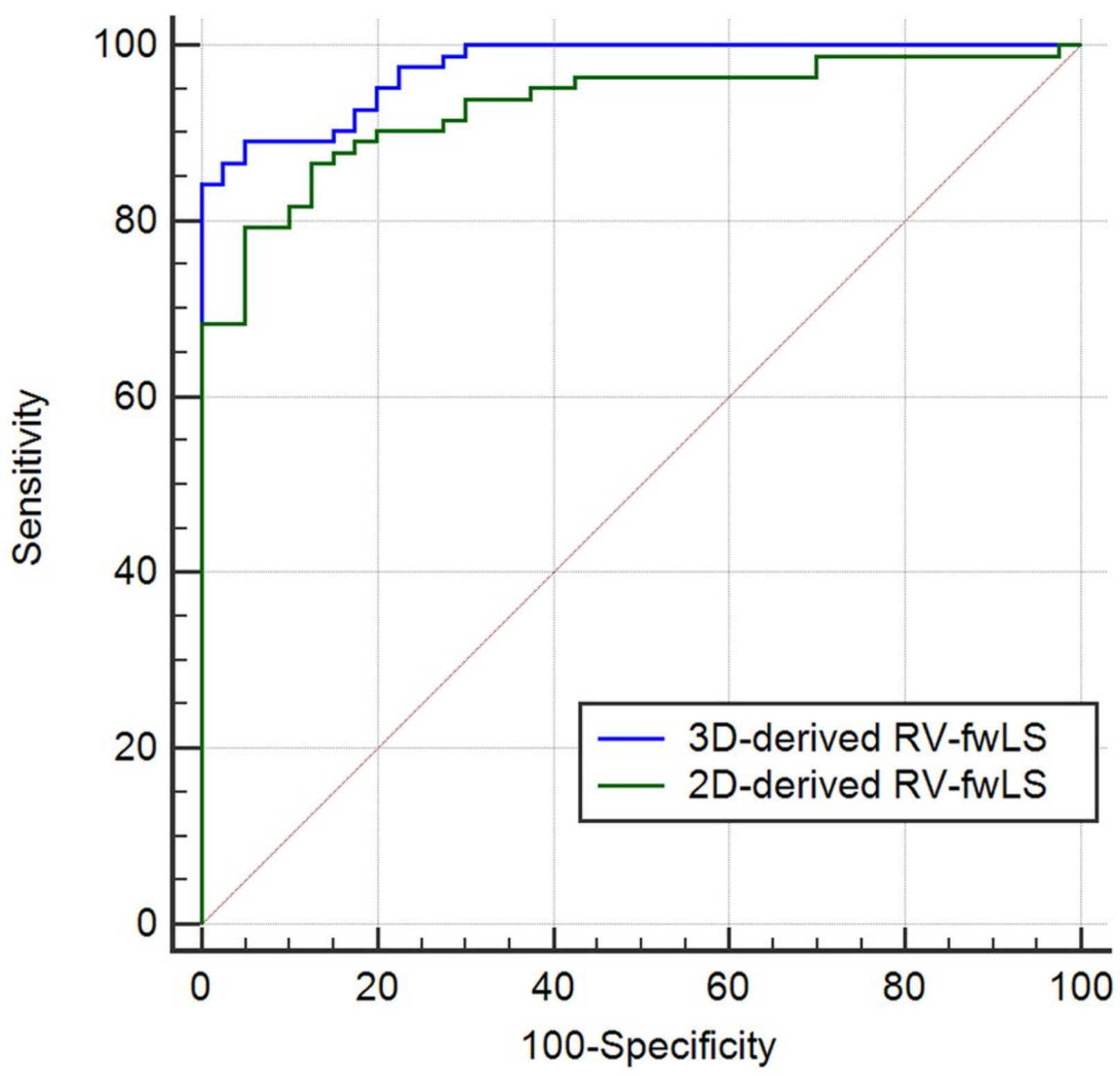

Figure 2

ROC analyses for the ability of 3DE- and 2DE-derived RV-fwLS to identify patients with RV dysfunction The analyses included all the study subjects $(n=122)$. 3DE $=$ three-dimensional echocardiography; $2 D E=$ two-dimensional echocardiography; RV-fwLS = peak systolic longitudinal strain in free wall of right ventricle. 

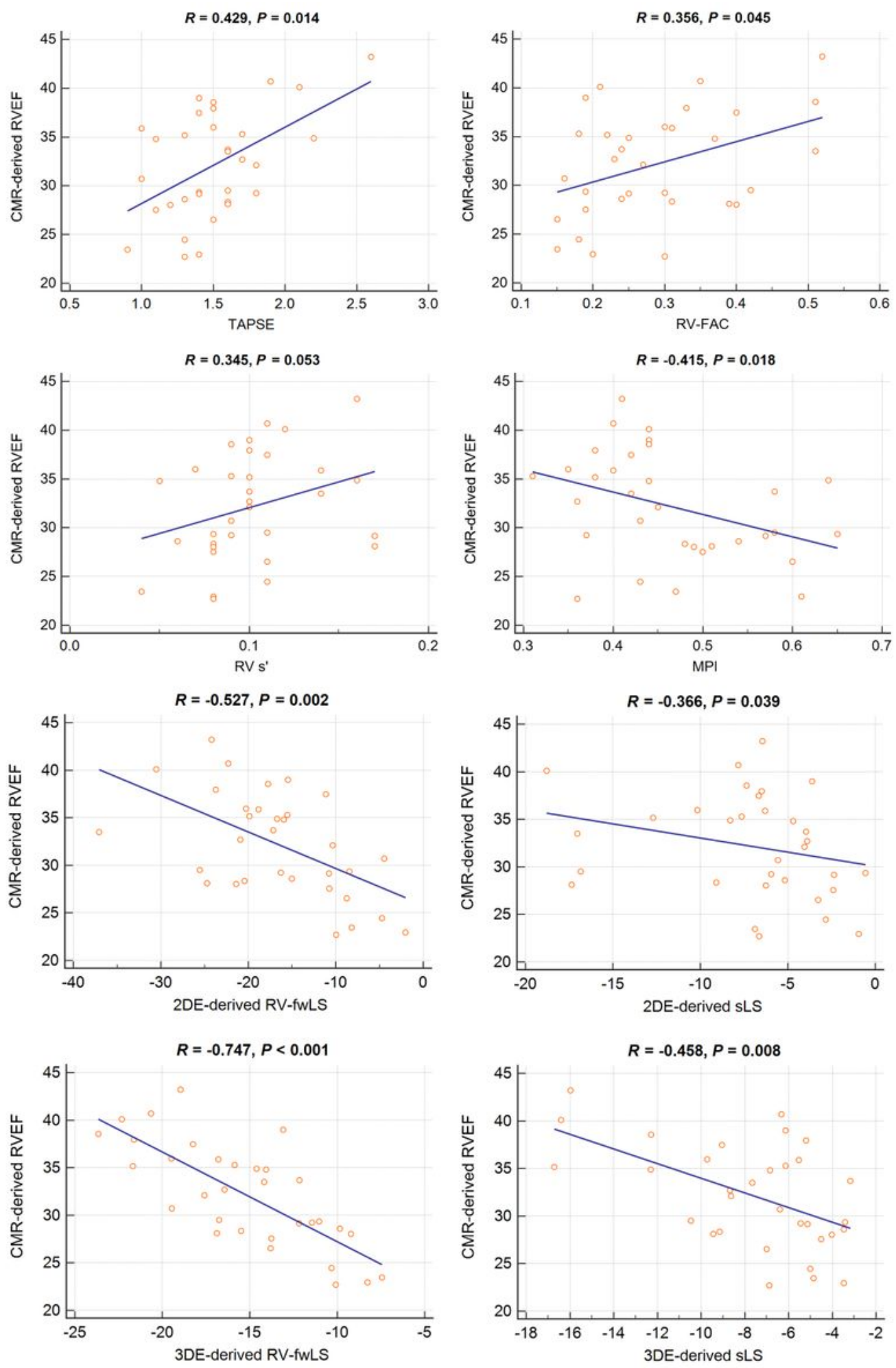

\section{Figure 3}

Correlation analyses between all measurements of RV systolic function with CMR-derived RVEF CMR = cardiac magnetic resounance; $E F$ = ejection fraction; TAPSE = tricuspid annular plane systolic excursion; $\mathrm{RV}=$ right ventricular; $\mathrm{FAC}=$ fractional area change; $\mathrm{s}^{\prime}=$ peak systolic velocity of tricuspid annular; $\mathrm{MPI}=$ myocardial performance index; $2 \mathrm{DE}=$ two-dimensional echocardiography; $3 \mathrm{DE}=$ three-dimensional 
echocardiography; RV-fwLS = peak systolic longitudinal strain in free wall of right ventricule; $s L S$ = peak systolic longitudinal strain in ventricular septum. $\mathrm{P}<0.05$ was considered significant.
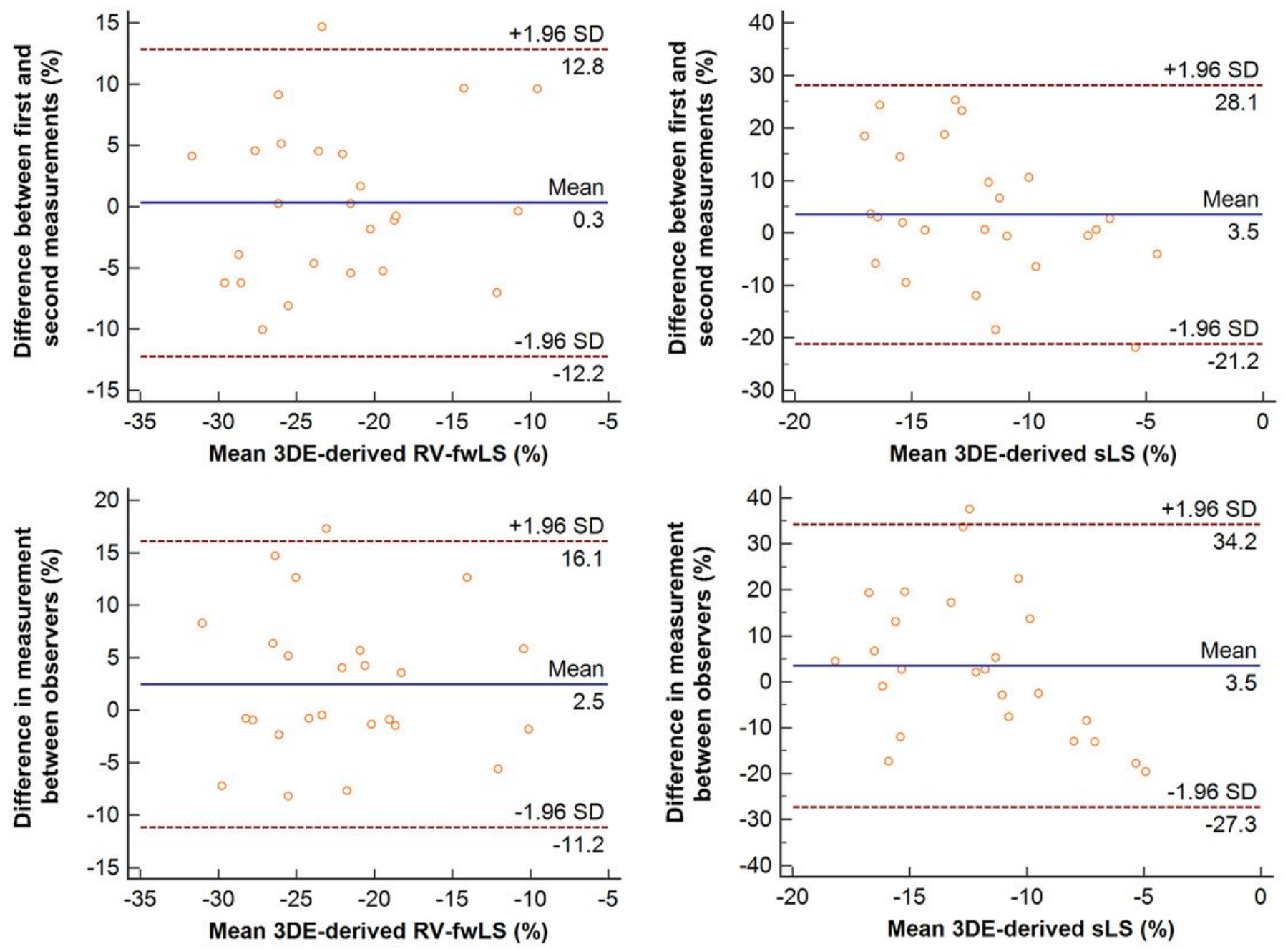

Figure 4

Bland-Altman agreement plots The figures exhibiting the mean differences and $95 \%$ limits of agreement for repeated measurements of 3DE-derived longitudinal strain of RV, performed by the same operator (intraobserver variability) or two independent operators (interobserver variability). All abbreviations are as shown in Figure 2. 